\title{
Agregação de valor à resíduo agroindustrial: remoção de fenol utilizando adsorvente preparado a partir de casca de amendoim
}

\author{
Thiago Silva ${ }^{1}$, Celmy Barbosa ${ }^{1}$, \\ Brigida Gama ${ }^{1}$, Graziele Nascimento ${ }^{1}$, Marta Duarte ${ }^{1}$
}

\author{
${ }^{1}$ Universidade Federal de Pernambuco, DEQ-UFPE, Avenida Artur de Sá, s/n, CEP: 50740-521 Recife, PE. \\ e-mail: thiago_pe@ig.com.br, celmy@ufpe.br, brigida.villar@hotmail.com, \\ grazielen@yahoo.com.br, mmmbduarte@gmail.com
}

\section{RESUMO}

Este trabalho teve como objetivo avaliar a eficiência do carvão ativado com $\mathrm{CO}_{2}\left(\mathrm{C}_{\mathrm{A}}\right)$ produzido a partir da casca de amendoim como adsorvente para remoção de fenol em solução aquosa (100-300 mg.L $\left.\mathrm{L}^{-1}\right)$. A caracterização do $\mathrm{C}_{\mathrm{A}}$ foi realizada pelos métodos de BET, DRX, TGA, FT-IR e $\mathrm{pH}_{\mathrm{pcz}}$, houve a caracterização da casca de amendoim $\left(\mathrm{C}_{\mathrm{S}}\right)$ para fins de comparação. Avaliou-se a influência do pH inicial da solução e a dosagem do adsorvente (D.A.). As condições de trabalho foram definidas com o auxílio de um planejamento fatorial $2^{3}$ (PF) avaliando a influência das variáveis: D.A., granulometria (G.) e velocidade de agitação (V.A.) sobre o processo adsortivo, tendo como resposta a capacidade adsortiva $q\left(\mathrm{mg} . \mathrm{g}^{-1}\right)$. Com base nos resultados do PF foram realizados estudos cinéticos e de equilíbrio de adsorção. O carvão foi classificado como mesoporoso e predominantemente amorfo. Na TGA foi observada maior estabilidade do material após a ativação. Nos espectros de FT-IR foram identificadas bandas referentes a grupos hidroxilas, carboxílicos e ésteres. O pH $\mathrm{pcz}_{\mathrm{p}}$ foi 10,4 possibilitando a utilização das soluções fenólicas em $\mathrm{pH}$ natural da solução. No PF, o maior $q$ foi obtido para o nível 4 g.L.-1 , G. < 0,090 mm e $300 \mathrm{rpm}$, condição adotada nos estudos cinéticos e de equilíbrio. A evolução cinética foi rápida até 120 minutos, atingindo o equilíbrio em torno de 480 minutos. O modelo pseudo $n$ ordem foi o que melhor se ajustou aos dados experimentais e foi constatado que o processo é controlado por mais de uma etapa, incluindo difusão intrapartícula. No estudo do equilíbrio os modelos que melhor se ajustaram aos dados experimentais foram os de Fritz-Schlunder e Redlich-Peterson apresentando $q_{\max }$ de $29,01 \pm 0,96 \mathrm{mg} \cdot \mathrm{g}^{-1}$. Os resultados demonstraram o potencial técnico do carvão em estudo para remoção de fenol presente em solução aquosa

Palavras-chave: ativação física, efluente industrial, resíduo agroindustrial.

\section{ABSTRACT}

This work had the aim of evaluate the efficiency of the activated carbon with $\mathrm{CO}_{2}\left(\mathrm{C}_{\mathrm{A}}\right)$ produced from peanut shell as adsorbent which was applied to remove phenol from aqueous water (100-300 mg. $\left.\mathrm{L}^{-1}\right)$. The characterization of the $\mathrm{C}_{\mathrm{A}}$ was performed by BET, DRX, TGA, FT-IR e $\mathrm{pH}_{\mathrm{pcz}}$ methods and was performed the characterization of the peanut shell $\left(\mathrm{C}_{\mathrm{S}}\right)$ for comparison. The influence of initial $\mathrm{pH}$ of the solution and the dosage of the adsorbent (D.A.) was studied. Working conditions were defined with the help of a $2^{3}$ factorial design (FD) evaluating the variables influence: DA, particle size (P.S) and stirring speed (S.S.) on the adsorption process, it is found as response the adsorption capacity, $q\left(\mathrm{mg} \mathrm{g}^{-1}\right)$. Based on the FD results kinetic studies and adsorption equilibrium were performed. The coal was classified as mesoporous and is predominantly amorphous. In the TGA greater stability of the material was observed after the activation. By FT-IR spectra bands were identified hydroxyl, carboxylic and ester groups. The $\mathrm{pH}_{\mathrm{pcz}}$ was 10.4 allowing the use of phenolic solutions in the natural $\mathrm{pH}$ of the solution. In FD the highest $q$ was obtained for the level 4 g.L-1, G. $<0.090$ $\mathrm{mm}$ and $300 \mathrm{rpm}$, adopted condition for executing the kinetic and equilibrium studies. The kinetic evolution was fast up to 120 minutes, reaching equilibrium around 480 minutes. The pseudo-order $n$ model was the best fit for experimental data and it was found that the process is controlled by more than one step, including intraparticle diffusion. For the equilibrium study the models that best fit the experimental data were FritzSchlunder and Redlich-Peterson with a $q_{\max }$ of $29.01 \pm 0.96 \mathrm{mg} \cdot \mathrm{g}^{-1}$. The results demonstrate the technical potential of the coal under study for the removal of phenol in aqueous solutions. 
Key words: agroindustrial residue, industrial effluent, physical activation.

\section{INTRODUÇÃO}

A maioria das indústrias são grandes consumidoras de água, consequentemente geram grandes volumes de efluentes que contêm compostos de difícil tratamento. Dentre estes compostos, estão os - fenólicos que são um dos principais poluentes encontrados nos efluentes de diversos tipos de indústrias, tais como: química, farmacêutica, corantes, pesticidas, petróleo e gás [1,2].

O fenol é considerado um poluente perigoso, afetando diretamente o meio ambiente e a saúde das pessoas. Por apresentarem alta toxicidade, os compostos fenólicos foram listados pela United States Environmental Protection Agency (USEPA) como poluentes prioritários e com indicação de monitoramento. Portanto, é imprescindível o tratamento dos efluentes industriais que contenham fenóis e seus derivados, antes do descarte em corpos hídricos [3,4].

Para o tratamento de efluentes industriais, existem diversas alternativas, como: processos oxidativos (biodegradação e fotocatálise) [5, 6] e processos adsortivos [7]. Dentre as alternativas existentes, os processos adsortivos se destacam por serem de simples aplicação e operação quando comparado a outros métodos de mesma eficiência, eficiência, disponibilidade de materiais adsorventes provenientes de resíduos, possibilidade de recuperação do adsorvato e do adsorvente, e o processo não ser afetado por substâncias tóxicas [8, 9].

A utilização de resíduos de biomassas para a produção de carvão é interessante por sua alta disponilidade, elevada área superficial, estrutura porosa bem desenvolvida e por ser um material renovável, sendo considerado eficiente por sua elevada capacidade de adsorção [10,11]. A ativação do carvão pode ser realizada por processos químicos ou processos físicos. $\mathrm{Na}$ ativação química são utilizados agentes ativadores, já na ativação física, são utilizados gases como $\mathrm{CO}_{2}$ e vapor d'água. No processo físico são utilizadas altas temperaturas, entretanto, em comparação com o químico, gera-se menos resíduos, provocando menor impacto ambiental $[12,13]$.

Algumas biomassas vêm sendo investigadas como adsorvente para a remoção de compostos fenólicos: resíduos agrícolas [14], caroço de abacate [15], casca de coco [16] e resíduo de Eucalyptus [17].

Dentre essas biomassas a casca de amendoim é um resíduo agroindustrial abundante, uma vez que, a produção de amendoim (Arachis hypogea) no Brasil é-foi em torno de 288,37 mil toneladas (em casca) na safra 2014/2015. Para esta produção, foi utilizada uma área territorial de 95,9 mil hectares [19]. A casca do amendoim representa $30 \%$ da produção do grão, normalmente utilizada como combustível para caldeira e alimento para gado. Entretanto nem toda casca produzida é utilizada, sendo necessária uma destinação adequada [20].

Diante do exposto, o objetivo deste trabalho foi preparar, caracterizar e avaliar um material adsorvente a partir da casca de amendoim,-para remoção de fenol presente em solução aquosa, avaliando a cinética e o equilíbrio de adsorção visando aplicação industrial.

\section{MATERIAIS E MÉTODOS}

Todos os ensaios foram realizados em erlenmeyers de $125 \mathrm{~mL}$ com tampa. Os experimentos de adsorção foram realizados em sistema de banho finito $\left(25 \pm 2^{\circ} \mathrm{C}\right)$ com auxílio de uma mesa agitadora (marca IKA, modelo KS 130 control). Ao final de cada ensaio as amostras foram filtradas, em papel de filtro faixa azul, para a quantificação do fenol, por meio do espectrofotômetro UV-Visível (marca Thermo Scientific, modelo Genesys 10S), no comprimento de onda de maior absorvância de $270 \mathrm{~nm}$, sendo o limite de detecção do método 0,09 $\mathrm{mg} \cdot \mathrm{L}^{-1}$, o limite de quantificação $0,3 \mathrm{mg} . \mathrm{L}^{-1}$ e a faixa linear de trabalho de 0,5 a $100 \mathrm{mg} . \mathrm{L}^{-1}$.

A concentração inicial da solução fenólica utilizada foi de $100 \mathrm{mg} . \mathrm{L}^{-1}$, baseada em estudos similares [1, 4]. A solução foi preparada a partir do fenol cristal p.a. (Ácido fênico, massa molecular 94,11 g.mol ${ }^{-1}$, marca DINÂMICA). Foram realizados ensaios em branco obedecendo rigorosamente o mesmo procedimento das amostras.

Foram utilizadas soluções de ácido clorídrico $\left(0,1 \mathrm{~mol} \cdot \mathrm{L}^{-1}\right)$ e hidróxido de sódio $\left(0,1 \mathrm{~mol} \cdot \mathrm{L}^{-1}\right)$ para o ajuste de $\mathrm{pH}$,- que foi medido com auxílio de um pHmetro (marca Hanna).

A quantidade adsorvida por massa de adsorvente $\left(q, \mathrm{mg} \cdot \mathrm{g}^{-1}\right)$ foi calculada utilizando a Equação 1.

$$
q_{e}=\frac{\left(C_{0}-C_{f}\right) V}{m}
$$


sendo: $C_{o}$ e $C_{f}$ as concentrações inicial e final, respectivamente (mg.L $\left.{ }^{-1}\right), V$ o volume da solução (L) e $m$-a massa de adsorvente $(\mathrm{g})$.

\subsection{Preparação do adsorvente}

A casca de amendoim $\left(\mathrm{C}_{\mathrm{s}}\right)$ foi triturada em liquidificador (Marca Mondial), lavada sucessivamente em água destilada, e seca em estufa a $105^{\circ} \mathrm{C}$ por 24 horas. A carbonização foi realizada em forno mufla (Marca Quimis) em rampa de aquecimento de 30 minutos a $100^{\circ} \mathrm{C}$, uma hora a $200^{\circ} \mathrm{C}$ e mais uma hora a $350^{\circ} \mathrm{C}$ sob atmosfera ambiente. Para a ativação do carvão, $20 \mathrm{~g}$ foram colocadas em um reator de quartzo que foi conectado a um forno elétrico (Marca Lindberg, Blue M). O material foi submetido a uma rampa de aquecimento de $10{ }^{\circ} \mathrm{C} . \mathrm{min}^{-}$ ${ }^{1}$, partindo da temperatura ambiente $\left(25 \pm 5^{\circ} \mathrm{C}\right)$ até $600^{\circ} \mathrm{C}$ em atmosfera inerte de nitrogênio seguido por fluxo de $\mathrm{CO}_{2}$ à vazão de $100 \mathrm{~mL} \cdot \mathrm{min}^{-1}$ por 1 hora cada. $\mathrm{O}$ carvão ativado $\left(\mathrm{C}_{\mathrm{A}}\right)$ foi classificado com uma série de peneiras de Tyler nas granulometrias $(\mathrm{G})<0,090 ; 0,090-0.150 ; 0,150-0,212 \mathrm{~mm}$.

\subsection{Caracterização do adsorvente}

A caracterização foi realizada para a casca de amendoim e para o carvão da casca de amendoim ativado com $\mathrm{CO}_{2}$ visando realizar uma comparação da estrutura dos dois materiais.

\subsubsection{Caracterização textural (BET)}

Para determinação da área superficial foi realizada a adsorção/dessorção de $\mathrm{N}_{2}$ a $77 \pm 5 \mathrm{~K}$ pelo método Brunauer-Emmett-Teller (BET) em um equipamento BELSORP-MINI da Bel Japan Inc. Para a determinação do diâmetro e do volume dos poros foi utilizado o método BJH. Foi realizado um pré-tratamento a $333 \mathrm{~K}$ sob vácuo por 3 horas a fim de remover a umidade da superfície da amostra.

\subsubsection{Difração de Raios-X (DRX)}

A análise foi realizada em Difratômetro de Raios-X BRUKER (modelo D8 ADVANCE) por meio de uma fonte de radiação de $\mathrm{Cu}-\mathrm{K} \alpha$ com voltagem de $30 \mathrm{kV}$ e corrente de $30 \mathrm{~mA}$. Os dados foram coletados na faixa de $2 \theta$ de $5^{\circ}$ a $80^{\circ}$ com passo de $0,05^{\circ}$ e passo no tempo de $2,0 \mathrm{~s}$.

\subsubsection{Análise Termogravimétrica (TGA)}

A análise foi realizada em uma termobalança (Perkin Elmer, modelo STA 6000), com taxa de aquecimento de $20{ }^{\circ} \mathrm{C} \cdot \mathrm{min}^{-1}$, sob fluxo de $20 \mathrm{~mL} \cdot \mathrm{min}^{-1}$ de $\mathrm{N}_{2}$. A massa de material utilizado nos cadinhos de platina foi fixada em $10 \mathrm{mg}$ e a-mesma foi aquecida de $30^{\circ} \mathrm{C}$ a $800^{\circ} \mathrm{C}$. Os resultados obtidos foram tratados com o auxílio do software Pyris Data Analysis, versão 11.

\subsubsection{Espectroscopia na região do infravermelho por transformada de Fourier (FT-IR)}

A análise foi realizada em um espectrômetro de marca Bruker Tensor 27, utilizando a técnica de Reflexão Total Atenuada (ATR). Os espectros de absorção foram obtidos na região do infravermelho de $4000 \mathrm{~cm}^{-1}$ a 600 $\mathrm{cm}^{-1}$ com resolução de $4 \mathrm{~cm}^{-1}$. Os resultados obtidos foram tratados através do software OPUS - Versão 7.2.

\subsubsection{Ponto de carga zero ( $\mathrm{pH}$ pcz)}

O ponto de carga zero foi realizado colocando-se $0,1 \mathrm{~g}$ do carvão em contato com água destilada em $\mathrm{pH}$ de 2 a 11 sob agitação constante de $300 \mathrm{rpm}$. Após $24 \mathrm{~h} \mathrm{o} \mathrm{pH}$ foi medido novamente e em seguida construído o gráfico $\left(\mathrm{pH}_{\text {final }}-\mathrm{pH}_{\text {inicial }}\right) v s$. $\mathrm{pH}_{\text {inicial }}$ O $\mathrm{pH}_{\mathrm{PCZ}} \mathrm{e}$ o valor do $\mathrm{pH}$ em que a curva intercepta o eixo do $\mathrm{pH}_{\text {incial }}$ [21].

\subsection{Processo adsortivo em banho finito}

\subsubsection{Avaliação do pH inicial da solução}


Foi avaliada a influência do $\mathrm{pH}$ inicial da solução fenólica no processo adsortivo através da realização de experimentos de adsorção em que $0,1 \mathrm{~g}$ do $\mathrm{C}_{\mathrm{A}}$ ficou em contato com $25 \mathrm{~mL}$ da solução fenólica $\left(100 \mathrm{mg} . \mathrm{L}^{-1}\right)$ no intervalo de $\mathrm{pH}$ variando de 2 a 10, permanecendo sob agitação constante de 300 rpm por 6 horas.

\subsubsection{Avaliação da dosagem do adsorvente}

Foi avaliada a influência da dosagem do $\mathrm{C}_{\mathrm{A}}$ em uma faixa de 4 a 40 g.L $\mathrm{L}^{-1}$ de solução, visando encontrar a melhor relação entre o percentual de remoção e a capacidade adsortiva para ser utilizado como ponto central no planejamento fatorial. Os ensaios foram realizados em erlenmeyers contendo $25 \mathrm{~mL}$ da solução fenólica na concentração de $100 \mathrm{mg} . \mathrm{L}^{-1}$ no pH definido no estudo anterior, a $300 \mathrm{rpm}$ por 6 horas.

\subsubsection{Definição da condição de trabalho}

Foi realizado um planejamento fatorial para avaliar as influências das variáveis: dosagem do adsorvente (D.A.) $\left(4,0 ; 8,0\right.$ e 1,2 g.L $\left.{ }^{-1}\right)$, granulometria do adsorvente (G.) (<0,090; 0,090-0,150 e 0,150-0,212 mm) e velocidade de agitação (V.A.) $(0 ; 150 ; 300 \mathrm{rpm})$ sobre o processo adsortivo. Nas condições avaliadas, foram utilizados 25 $\mathrm{mL}$ da solução de $100 \mathrm{mg} . \mathrm{L}^{-1}$ de fenol.

Foi utilizado o planejamento fatorial $2^{3}$ com ponto central em triplicata, resultando em 11 experimentos. Os ensaios foram realizados em ordem aleatória e a eficiência do processo foi determinada a partir da capacidade de adsorção $\left(q, \mathrm{mg} \cdot \mathrm{g}^{-1}\right)$.

Os cálculos dos efeitos dos fatores e as interações entre eles com os seus respectivos erros padrão foram realizados segundo [22], com auxilio do programa Statistica for Windows 6.0.

\subsubsection{Estudo cinético e de equilíbrio de adsorção}

Os estudos, cinético e de equilíbrio, foram realizados nas condições operacionais definidas pelo planejamento fatorial.

Para o estudo cinético, os tempos foram de 1-600 min, em que o adsorvente ficou em contato com a solução fenólica a $100 \mathrm{mg} \cdot \mathrm{L}^{-1}$.

Foram utilizados os modelos cinéticos de pseudo-primeira ordem (Equação 2), pseudo-segunda ordem (Equação 3) e pseudo $n$ ordem (Equação 4), além do modelo de difusão intrapartícula de Weber-Morris (Equação 5) para avaliação do mecanismo de adsorção.

Modelo de pseudo primeira ordem - Uma análise da cinética de adsorção é realizada pela equação de Lagergren (1898) de pseudo primeira ordem (Equação 2), baseada na capacidade de adsorção proporcional ao número de sítios livres dos sólidos [23].

$$
\frac{d q_{t}}{d t}=k_{1}\left(q_{e}-q_{t}\right)
$$

em que: $k_{l}$, a constante da taxa de adsorção de pseudo primeira ordem $\left(\mathrm{min}^{-1}\right), q_{e}$, a capacidade adsortiva no equilíbrio, $q_{t}$, a capacidade adsortiva no tempo (mg.g ${ }^{-1}$ ), e $t$; o tempo (minutos).

Modelo de pseudo segunda ordem - O modelo de pseudo segunda ordem assume que a adsorção química controla o processo, sendo dependente das interações físico-químicas entre adsorvente/adsorvato livres [23]. Esse modelo pode ser representado de acordo com a Equação 3:

$$
\frac{d q_{t}}{d t}=K_{2}\left(q_{e}-q_{t}\right)^{2}
$$


sendo: $k_{2}$ é a constante da taxa de adsorção de pseudo segunda ordem $\left(\mathrm{g} \cdot \mathrm{mg}^{-1} \cdot \mathrm{min}^{-1}\right), q_{e}$ e $q_{t}$ são as capacidades adsortivas no equilíbrio e ao longo do tempo $\left(\mathrm{mg} \cdot \mathrm{g}^{-1}\right)$, respectivamente.

Modelo de pseudo $n$ ordem - O modelo de pseudo $n$ ordem explica o comportamento intermediário do processo de adsorção entre as curvas de pseudo primeira ordem (Equação 2) e pseudo segunda ordem (Equação 3), valor de $n$ entre 1 e 2. Quando $n$ for maior do que 2, as curvaturas das isotermas aumentam com o aumento da ordem $n$ [24]. O modelo de pseudo $n$ ordem pode ser expresso de acordo com a Equação 4 :

$$
\frac{d q_{t}}{d t}=K_{n}\left(q_{e}-q_{t}\right)^{n_{o}}
$$

em que: $K_{n}$, a constante da velocidade de reação do modelo pseudo $n$ ordem e $n_{o}$; a ordem de reação $\left(\mathrm{g}^{\mathrm{n}-1} \mathrm{mg}^{1-}\right.$ $\left.{ }^{\mathrm{n}} \min ^{-1}\right)$;

Modelo de difusão intrapartícula - Se a difusão intrapartícula for o fator determinante da velocidade, a remoção do adsorvato variará em relação à raiz quadrada do tempo. Assim, o coeficiente de difusão intrapartícula $\left(k_{d i f}\right)$ pode ser obtido de acordo com a Equação 5.

$$
q_{t}=k_{d i f} t^{1 / 2}+C
$$

sendo: $k_{d i f}$ é a constante de difusão intrapartícula $\left(\mathrm{mg} \cdot \mathrm{g}^{-1} \cdot \mathrm{min}^{-1 / 2}\right)$ e $C$ é uma constante relacionada com a resistência à difusão $\left(\mathrm{mg} \cdot \mathrm{g}^{-1}\right)$ [25].

Para o estudo de equilíbrio foram realizados experimentos no tempo estabelecido no estudo cinético, em que o adsorvente permaneceu em contato com solução fenólica nas concentrações de $5 ; 15 ; 35 ; 50 ; 75$; $100 ; 120 ; 150 ; 200 ; 300 \mathrm{mg} . \mathrm{L}^{-1}$.

Para avaliar os dados do equilíbrio do processo, foram aplicados os modelos de adsorção de Langmuir (Equação 6), Freundlich (Equação 7), Fritz-Schlunder (Equação 8) e Redlich-Peterson (Equação 9), conforme [26].

O modelo da isoterma de Langmuir para um sistema monocomponente e homogêneo é baseado na hipótese de adsorção de moléculas na superfície de um adsorvente, sendo representado pela Equação 6 [27]:

$$
q_{e}=\frac{q_{\max } K_{L} C_{e}}{1+K_{L} C_{e}}
$$

sendo: $q_{\max }, o$ parâmetro de que expressa a capacidade máxima adsortiva $\left(\mathrm{mg} \mathrm{g}^{-1}\right), K_{L}$; a constante de adsorção de equilíbrio, que representa a afinidade entre o adsorvente e adsorvato $\left(\mathrm{L}_{\mathrm{g}} \mathrm{g}^{-1}\right.$ ) e $C_{e}$; a concentração do adsorvato no equilíbrio (mg. $\left.\mathrm{L}^{-1}\right)$.

A isoterma de Freundlich considera que a adsorção ocorre em uma superfície heterogênea e basea-se na existência de uma estrutura em várias camadas, prevendo uma distribuição exponencial de vários sítios de adsorção com energias diferentes. À medida que a adsorção ocorre, a energia de adsorção diminui, portanto a equação de Freundlich (Equação 7) pode ser aplicada à superfícies não uniformes [28].

$$
q_{e}=K_{F}\left(C_{e}\right)^{\frac{1}{n}}
$$

em que: $K_{F}\left(\mathrm{mg}_{\mathrm{g}} \mathrm{g}^{-1}\right)$ e $n$ (adimensional) são constantes do modelo de Freundlich. $K_{F}$ indica a capacidade da adsorção do adsorvente e $n$ indica a eficiência do processo de adsorção. Para o valor da constante $n$ entre $1 \mathrm{e}$ 10, a adsorção é favorável.

O modelo da isoterma de Fritz-Schlunder (Equação 8) possui quatro parâmetros e foi derivado da 
equação de Langmuir-Freundlich. Em concentrações elevadas, o modelo se limita à equação de Freundlich; e quando $b_{1}$ e $b_{2}$ são iguais a 1, o modelo se reduz à equação de Langmuir [29].

$$
q_{e}=\frac{K_{F S} C_{e}^{b_{1}}}{1+a C_{e}^{b_{2}}}
$$

sendo: $K_{F S}$, a constante de Fritz-Schlunder, e $b_{1}$ e $b_{2}$, os fatores de heterogeneidade.

O modelo da isoterma de Redlich-Peterson possui três parâmetros, sendo $g$ adimensional, Equação 9 $[26]$

$$
q_{e}=\frac{A C_{e}}{1+B C_{e}^{g}}
$$

em que: $A, B$ e $g$ são constantes do modelo de Redlich-Peterson.

Para verificar o desempenho dos modelos avaliados para um nível de $95 \%$ de confiança, foi utilizado o Teste $F$, que compara a precisão entre métodos de acordo com [30].

\section{RESULTADOS E DISCUSSÕES}

\subsection{Caracterização do adsorvente}

Além da caracterização do carvão ativado com $\mathrm{CO}_{2}\left(\mathrm{C}_{\mathrm{A}}\right)$ também foi realizada a caracterização para a casca de amendoim $\left(\mathrm{C}_{\mathrm{s}}\right)$ para fins de comparação.

\subsubsection{Caracterização textural (BET)}

Os resultados da caracterização textural através da adsorção/dessorção de $\mathrm{N}_{2}$, para a casca de amendoim e para o carvão da casca de amendoim ativado com $\mathrm{CO}_{2}$ estão apresentados na Tabela 1.

\begin{tabular}{|c|c|c|c|}
\hline MATERIAL & $\begin{array}{l}\text { ÁREA SUPERFICIAL } \\
\qquad\left(\mathrm{m}^{2} \mathrm{~g}^{-1}\right)\end{array}$ & $\begin{array}{l}\text { VOLUME TOTAL DOS POROS } \\
\left(\mathrm{cm}^{3} \mathrm{~g}^{-1}\right)\end{array}$ & $\begin{array}{c}\text { DIÂMETRO DOS POROS } \\
(\AA \AA)\end{array}$ \\
\hline $\mathrm{C}_{\mathrm{S}}$ & 54,5 & 0,07 & 46,3 \\
\hline $\mathrm{C}_{\mathrm{A}}$ & 402,6 & 0,20 & 40,1 \\
\hline
\end{tabular}

Tabela 1: Resultados obtidos por adsorção/dessorção de $\mathrm{N}_{2}$ para os materiais.

De acordo com [31], materiais com diâmetro de poros entre 20-500 A, são classificados como mesoporosos, sendo assim os materiais avaliados neste trabalho (Tabela 1) estão classificados nessa faixa. Como diâmetro do poro do $\mathrm{C}_{\mathrm{A}}(40,1$ Å) é muito maior que o diâmetro da molécula de fenol $(6,2 \AA$ A é possível o acesso aos sítios disponíveis para adsorção.

Resultados semelhantes foram encontrados por [15] que utilizaram caroço de abacate para produção de carvão obtendo adsorventes mesopororos. Estudo mais recente desenvolvido por [33], utilizando ativação física na preparação de adsorvente, a partir do resíduo de madeira, observaram área superficial de $409 \mathrm{~m}^{2} \cdot \mathrm{g}^{-1} \mathrm{e}$ do volume total dos poros igual a $0,21 \mathrm{~cm}^{3} \cdot \mathrm{g}^{-1}$.

Analisando a Tabela 1, observa-se um aumento na área superficial e no volume total dos poros para o carvão ativado. Conforme [32] a ativação abre os poros que se encontravam inacessíveis durante o processo de carbonização, além disso, cria-se novos poros e ainda aumenta-se os poros já existentes, facilitando a eliminação das impurezas presentes no material.

As isotermas de adsorção e dessorção para os materiais estão apresentadas na Figura 1. 

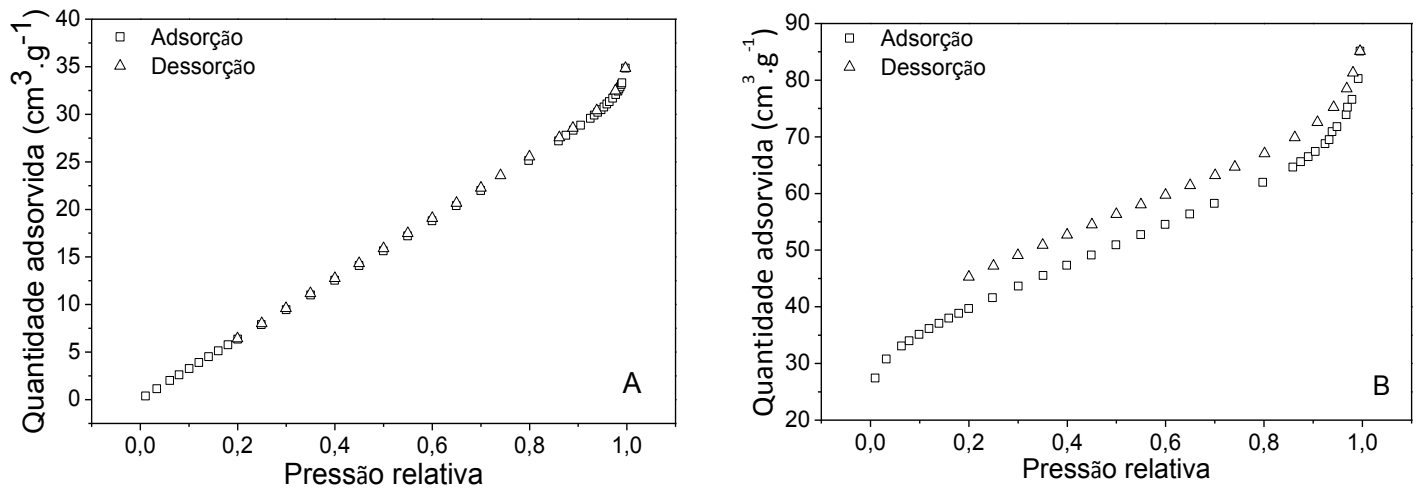

Figura 1: Isotermas de adsorção e dessorção da $C_{S}(A)$ e do $C_{A}(B)$.

É possível observar na Figura 1, na parte inicial, isoterma do tipo I, que é característica de materiais microporosos. Entretanto, com o aumento da pressão relativa, para ambos os materiais, pode-se observar isotermas do tipo IV, que indicam transição de microporosidade para mesoporosidade. Esta transição ocorre principalmente devido ao fenômeno de histerese, que pode ser observado com maior evidencia na Figura 1B, sugerindo uma maior dispersão no volume dos poros. A histerese ocorre quando o processo de evaporação é diferente do processo de condensação. Segundo [35], tal fenômeno está associado à condensação capilar que justificaria o aumento da adsorção em materiais mesoporosos, quando comparados a sólidos não porosos.

Zhong et al. [36] utilizaram a adsorção de nitrogênio para determinação da porosidade do carvão da casca de amendoim ativado quimicamente. Observaram isotermas mistas do tipo I e IV, que sugerem que o material possuía características de sólido microporoso com desenvolvimento considerável de material mesoporoso, resultados semelhantes ao obtido neste trabalho com ativação física.

\subsubsection{Difração de raios-X (DRX)}

O difratograma de raios-X para a casca de amendoim e para o carvão da casca de amendoim ativado pode ser observado na Figura 2.

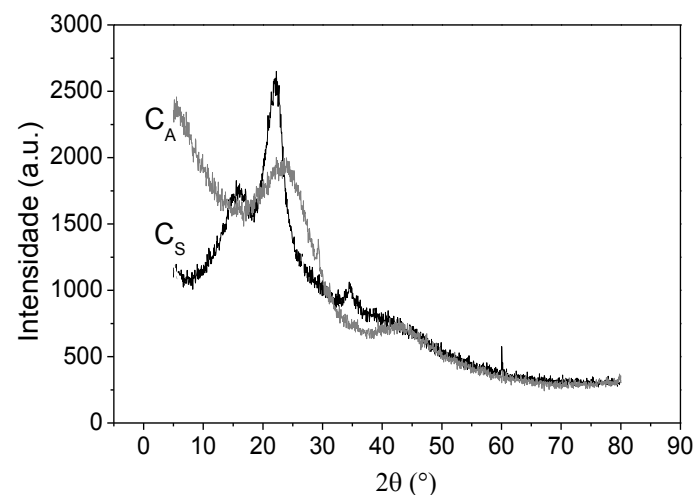

Figura 2: Difratograma de Raios- $\mathrm{X}$ para $\mathrm{C}_{\mathrm{S}}$ e $\mathrm{C}_{\mathrm{A}}$.

Através do difratograma pode ser observada a presença picos largos na região $23^{\circ}$ e $43^{\circ}$, para o carvão ativado, já para a casca de amendoim apenas na região de $23^{\circ}$. Picos nas regiões $23^{\circ}$ e $43^{\circ}$ indicam a formação de uma reduzida estrutura cristalina de carbono correspondente às reflexões da estrutura de microcristais tipo grafite, conforme [37]. A estrutura cristalina do carvão favorece a interação entre a ligação dos elétrons do sistema- $\pi$ de anéis aromáticos, como o do fenol, com o sistema- $\pi$ da camada grafite [38]. Observa-se também que as curvas não apresentam picos acentuados, característica típica de material que possui estrutura amorfa de acordo com [39].

\subsubsection{Análise termogravimétrica (TGA)}


Os resultados das análises termogravimétricas estão apresentados na Figura 3.
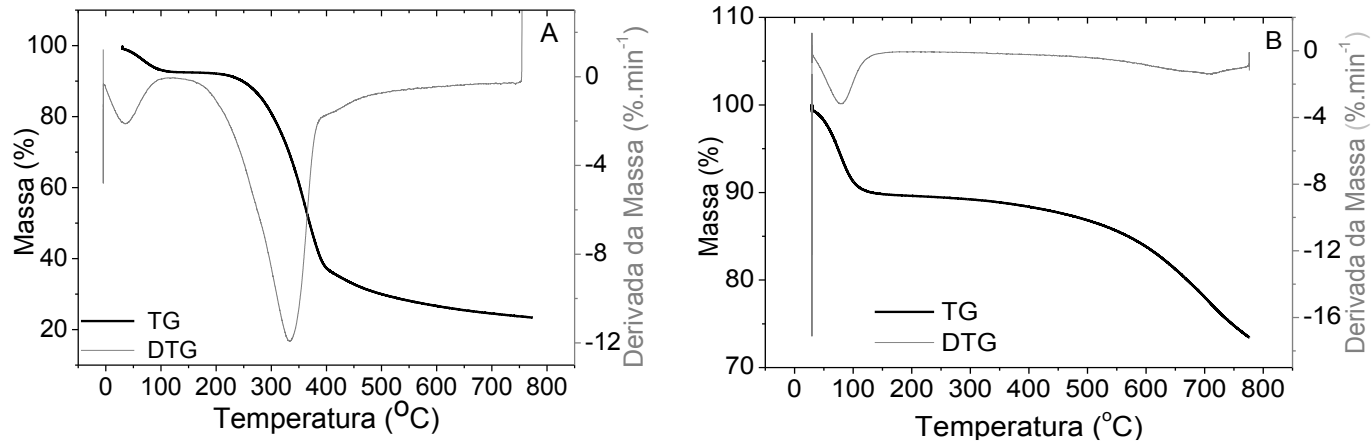

Figura 3: Análise termogravimétrica para $\mathrm{C}_{\mathrm{S}}(\mathrm{A})$ e para $\mathrm{C}_{\mathrm{A}}(\mathrm{B})$.

O comportamento termogravimétrico da casca de amendoim $\left(\mathrm{C}_{\mathrm{S}}\right)$ (Figura $3 \mathrm{~A}$ ) apresentou a primeira perda de massa em até $120^{\circ} \mathrm{C}$ provavelmente por causa à eliminação de umidade. A segunda perda ocorreu entre 200 e $500^{\circ} \mathrm{C}$, provavelmente devido à decomposição de lignina, estrutura com maior estabilidade a perda de grupos carboxílicos $[16,40]$. A partir de $400^{\circ} \mathrm{C}$ a massa foi reduzida para aproximadamente $25 \%$ do total de sua massa inicial, demonstrando estabilidade térmica em torno de $600^{\circ} \mathrm{C}$, sendo esta a temperatura utilizada no processo de ativação.

Para $\mathrm{C}_{\mathrm{A}}$ (Figura 3B), pode-se observar duas perdas de massa. A primeira perda de massa ocorreu abaixo de $120^{\circ} \mathrm{C}$, provavelmente em consequência da perda de umidade e/ou água de constituição. A segunda perda de massa acima de $500^{\circ} \mathrm{C}$, presumivelmente por efeito da lenta decomposição de frações de uma estrutura mais complexa, possivelmente a lignina presente em materiais de origem vegetal. $\mathrm{O} \mathrm{C}_{\mathrm{A}}$ reduziu sua massa até, aproximadamente, $75 \%$ do valor inicial, demonstrando maior estabilidade térmica do que o $\mathrm{C}_{\mathrm{s}}$.

Comportamentos semelhantes foram observados por [40] que realizaram estudos termogravimétricos do carvão ativado preparado a partir do pinhão manso.

\subsubsection{Espectroscopia na região do infravermelho por transformada de Fourier (FT-IR)}

O gráfico da espectroscopia na região do infravermelho esta apresentado na Figura 4.

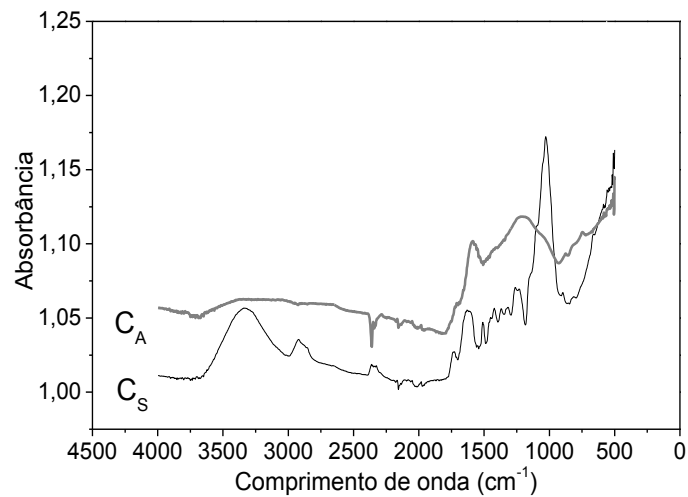

Figura 4: Espectros de infravermelhos para $C_{S}$ e $C_{A}$.

A banda entre 3200 e $3550 \mathrm{~cm}^{-1}$, Figura 4, para $\mathrm{C}_{\mathrm{S}}$, é atribuída ao alongamento vibracional de hidroxilas em grupos funcionais de álcool ou fenol, entretanto para o $\mathrm{C}_{\mathrm{A}}$ essas vibrações foram enfraquecidas provavelmente devido à degradação de material celulósico e lignocelulósico presentes em materiais de origem vegetal como a casca de amendoim. As bandas localizadas entre $1620 \mathrm{~cm}^{-1}$ e $1680 \mathrm{~cm}^{-1}$ são de ligação dupla entre carbonos, podendo ser observadas nos dois materiais. Este alongamento é pertencente ao grupo alcenila, presente em materiais como a lignina conforme [41] e [36].

Observou-se ainda que a banda localizada entre 2853 e $2962 \mathrm{~cm}^{-1}$ para a casca de amendoim $\mathrm{C}_{\mathrm{S}}$ é atribuída provavelmente ao alongamento vibracional $\mathrm{CH}$ pertencente ao grupo alquila, o qual desapareceu para 
o $C_{A}$, indicando que o hidrogênio foi removido após a carbonização [41].

Segundo [36], os picos simultâneos localizados em $2362 \mathrm{~cm}^{-1}, 1570 \mathrm{~cm}^{-1}$ e $1215 \mathrm{~cm}^{-1}$ no espectro da casca de amendoim $\left(\mathrm{C}_{\mathrm{A}}\right)$ também sugerem que a presença de grandes quantidades de material lignocelulósico.

A banda na região de $1223-1000 \mathrm{~cm}^{-1}$ do $\mathrm{C}_{\mathrm{A}}$ indica a existência de uma ligação simples C-O característica de grupos funcionais carboxílicos, álcoois, fenóis e ésteres de acordo com [42]. Este alongamento vibracional foi enfraquecido após a ativação, indicando uma possível degradação da estrutura do material com o aumento da temperatura.

A diminuição dos picos e bandas referentes aos grupos funcionais observadas no FT-IR, após a ativação, provavelmente está relacionada com a eliminação de grupos oxigênio da casca de amendoim durante esse processo. Tal diminuição de grupos funcionais sugere uma maior estabilidade térmica vinculada à degradação do material lignocelulósicos com o aumento da temperatura. Esta maior estabilidade térmica do carvão ativado é evidenciada na análise termogravimétrica por consequência a sua menor perda de massa.

Conforme [38] e [43] o processo de adsorção do fenol na superfície do carvão ativado está relacionado com os grupos superficiais, principalmente a carbonila, que interagem com o grupo hidroxila do fenol. As camadas grafíticas ficam num plano de base, enquanto a molécula de fenol fica ligada perpendicularmente.

\subsubsection{Ponto de carga zero ( $\left.\mathrm{pH}_{\mathrm{pcz}}\right)$}

$\mathrm{O}$ ponto de carga zero para a casca de amendoim e para o carvão da casca de amendoim ativado com $\mathrm{CO}_{2}$ pode ser observado na Figura 5.

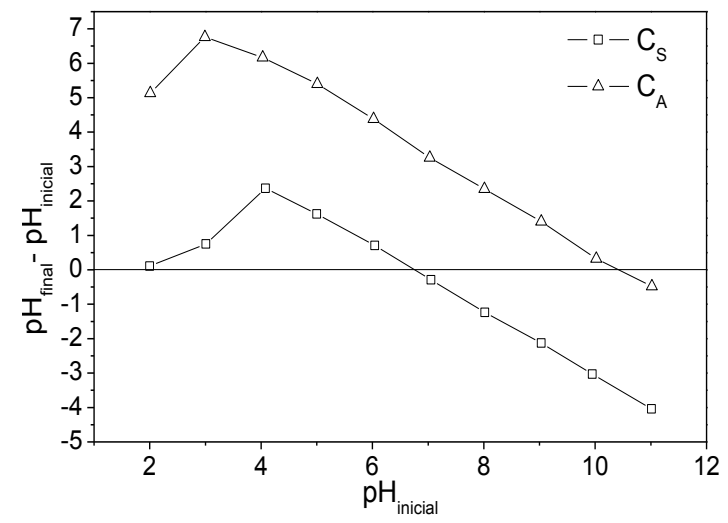

Figura 5: Ponto de carga zero para $\mathrm{C}_{\mathrm{S}}$ e $\mathrm{C}_{\mathrm{A}}$.

Conforme Figura 5, a intercessão da curva com o eixo horizontal da escala de $\mathrm{pH}_{\text {inicial }}$, ocorreu num valor de $\mathrm{pH}$ igual 6,7 para $\mathrm{C}_{\mathrm{S}}$ e 10,4 para $\mathrm{C}_{\mathrm{A}}$, que corresponde ao $\mathrm{pH}$ do ponto de carga zero. Este aumento do $\mathrm{pH}_{\mathrm{PCZ}}$ indica um aumento de cargas positivas na superfície do material após a ativação, favorecendo a adsorção de compostos como o fenol. De acordo com [44] este aumento de cargas positivas, sugere que, durante o processo de ativação, o $\mathrm{CO}_{2}$ reagiu com o carvão, liberando óxidos de carbono presentes na superfície do carvão. Esta liberação de óxidos pode ser observada, também, na espectroscopia de infravermelho, sugerindo a eliminação de grupos oxigênio.

Este resultado coincide com o de [45], utilizando resíduo de bagaço de mandioca sem modificação, que obtiveram pHpcz igual a 6,17 e [15] que encontraram $\mathrm{pH}_{\mathrm{pcz}}$ de 9,7 para carvão do caroço de abacate ativado com $\mathrm{CO}_{2}$.

De acordo com os resultados obtidos, quando comparado à casca de amendoim, o $\mathrm{C}_{\mathrm{A}}$ apresentou características que favorecem a adsorção de compostos como o fenol, sendo assim este foi utilizado para os estudos de adsorção.

\subsection{Processo adsortivo em banho finito}

O processo de adsorção em sistema de banho finito foi realizado para o carvão da casca de amendoim ativado com $\mathrm{CO}_{2}$. 


\subsubsection{Avaliação do pH inicial da solução}

Os resultados obtidos para a avaliação do efeito do pH inicial da solução fenólica estão apresentados na Figura 6.

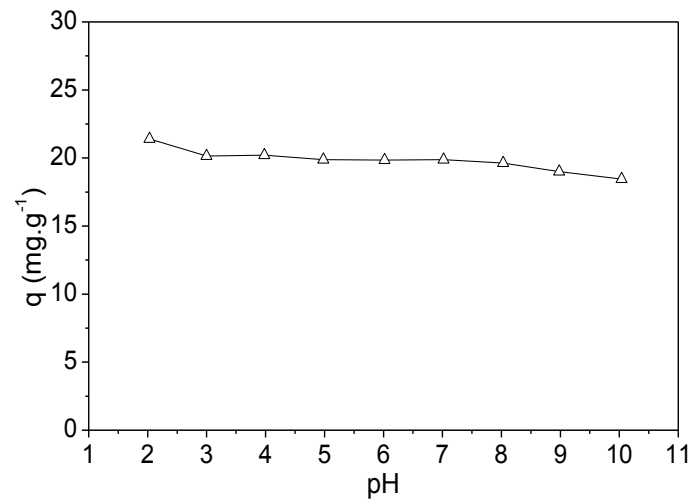

Figura 6: Influência do pH inicial da solução fenólica no processo adsortivo $\left(\mathrm{C}_{\mathrm{A}}\right) \cdot \mathrm{C}_{\mathrm{o}}=100 \mathrm{mg} \cdot \mathrm{L}^{-1}$; G. 0,150-0,212 mm; $\mathrm{t}=6 \mathrm{~h} ; \mathrm{V} . \mathrm{A} .300 \mathrm{rpm}$.

Como pode ser observado na Figura 6, o pH inicial da solução fenólica teve baixa influência sobre a capacidade adsortiva, provavelmente porque os valores estudados encontravam-se abaixo do $\mathrm{pH}_{\mathrm{pcz}}$. Nesta faixa, a superfície do carvão possui uma maior quantidade de cargas positivas, favorecendo a adsorção de compostos como o fenol. Nesta faixa, a superfície do carvão possui uma maior quantidade de cargas positivas, favorecendo a adsorção de compostos como o fenol.

$\mathrm{O} \mathrm{pH}$ utilizado neste trabalho para os demais estudos foi 6 , pois é o $\mathrm{pH}$ natural da solução fenólica preparada e menor que o $\mathrm{pH}_{\mathrm{pcz}}$.

\subsubsection{Avaliação da dosagem do adsorvente}

O efeito da variação da dosagem do adsorvente na adsorção de fenol pode ser observado na Figura 7.

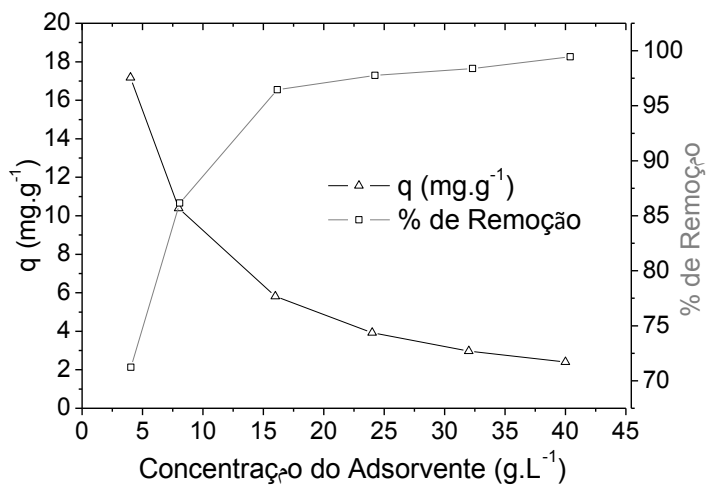

Figura 7: Influência da concentração para $C_{A} \cdot C_{0}=100 \mathrm{mg} \cdot \mathrm{L}^{-1}$; G. 0,150-0,212 mm; t = 6h; V.A. $300 \mathrm{rpm}$.

Conforme a Figura 7, o percentual de remoção de fenol aumenta com o aumento da concentração do adsorvente de 71,2 para 99,5\%. Este aumento, provavelmente ocorreu devido a uma maior disponibilidade de área superficial e consequentemente do número de sítios ativos, conforme [46]. Já a capacidade adsortiva ( $q$, $\mathrm{mg} . \mathrm{g}^{-1}$ ) diminui, de $17,2 \mathrm{mg} \cdot \mathrm{g}^{-1}$ para $2,4 \mathrm{mg} \cdot \mathrm{g}^{-1}$, tendo em vista a redução da razão adsorvato/adsorvente.

A dosagem do adsorvente de $8 \mathrm{~g} . \mathrm{L}^{-1}$ foi utilizada como ponto central para o planejamento fatorial por apresentar melhor relação entre porcentagem de remoção do fenol e capacidade adsortiva do carvão, esta dosagem do adsorvente corresponde a interseção entre as duas curvas.

\subsubsection{Definição da condição de trabalho}


Com os resultados dos ensaios do planejamento fatorial os efeitos principais e suas interações de 2 e 3 fatores, com seus respectivos erros padrão $(s)$, foram calculados utilizando-se o programa Statistica for Windows 6.0. para um nível de $95 \%$ de confiança.

Apenas a interação dosagem do adsorvente e granulometria $\left((1)^{*}(2)\right)$ não foi estatisticamente significativa para um nível de $95 \%$ de confiança, nas condições estudadas. Este resultado pode ser observado através da carta de Pareto na Figura 8, uma vez que não ultrapassou o p igual 0,05.

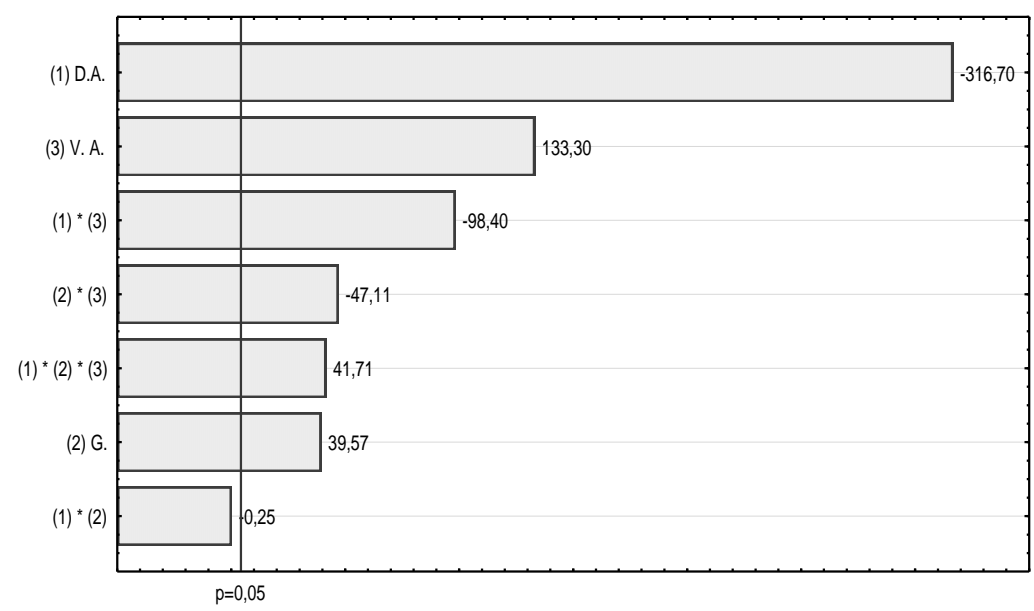

Figura 8: Carta de Pareto dos efeitos calculados $\left(\mathrm{C}_{\mathrm{A}}\right)$ (erro puro de 0.002).

Como se obtiveram efeitos de interações significativos, observados na carta de Pareto (Figura 8), é importante avaliar também os gráficos de superfície de resposta.

Na Figura 9, pode-se observar as superfícies de resposta referentes às interações de dois fatores estatisticamente significativos, mostrando a influência das variáveis nos níveis estudados.

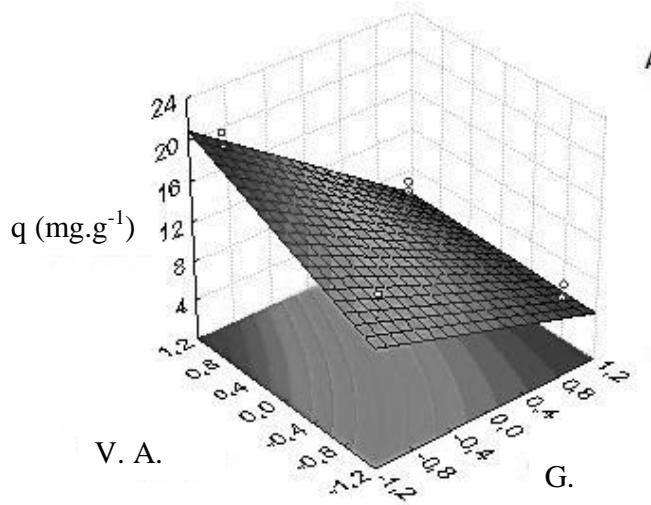

A

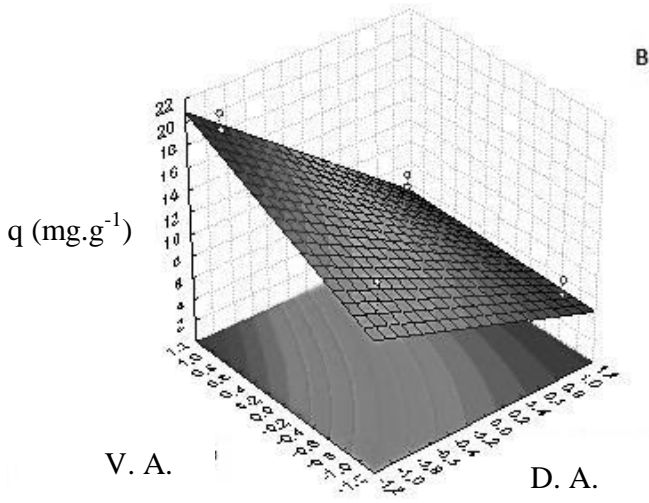

Figura 9: Superfícies de resposta para a capacidade adsortiva. (A) V.A. vs. C.A.; (B) V.A. vs. G.

Observa-se na Figura 9, que a maior capacidade adsortiva foi obtida para menor granulometria e concentração do adsorvente e para maior velocidade de agitação.

Segundo [27], partículas de menor granulometria se mostram mais eficientes devido ao aumento da área superficial sem uma alta resistência a transferência de massa, favorecendo o processo de adsorção.

A capacidade adsortiva aumentou com o aumento da velocidade de agitação. Segundo [47], isto ocorre provavelmente devido à resistência à transferência de massa externa se tornar desprezível quando a velocidade de agitação é suficiente para minimizar a espessura da camada limite que envolve a partícula sólida.

A partir das interações, pode-se observar que a maior quantidade de fenol adsorvida por massa de adsorvente, nos níveis avaliados, foi obtida para 4 g.L $\mathrm{L}^{-1}$, V.A. $300 \mathrm{rpm}$ e G. $<0,090 \mathrm{~mm}$. 


\subsubsection{Estudo cinético e de equilíbrio de adsorção}

Pode ser observada, na Figura 10, a curva de evolução cinética da adsorção do fenol e os ajustes dos modelos cinéticos de pseudo-primeira ordem, pseudo-segunda ordem e pseudo $n$ ordem para o carvão em estudo.

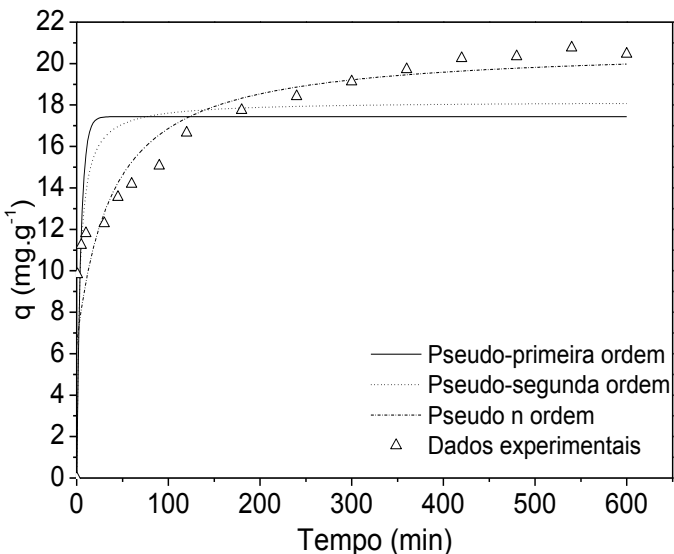

Figura 10: Evolução cinética da adsorção do fenol e os ajustes não lineares dos modelos cinéticos. $\mathrm{pH}=6,0, \mathrm{C}_{0}$ de 100 mg.L $\mathrm{L}^{-1}, \mathrm{C}_{\mathrm{ads}}$ de $4 \mathrm{~g} \mathrm{~L}^{-1}$, G. $<0,090 \mathrm{~mm}$ e V.A. $300 \mathrm{rpm}$.

A evolução cinética inicial foi acentuada, com a maior remoção em até 120 minutos, provavelmente devido a uma maior quantidade de sítios livres disponíveis. A etapa seguinte foi lenta por causa à diminuição destes sítios livres à medida que o fenol é adsorvido, atingindo o equilíbrio em torno de 480 minutos, como se observa na Figura 10.

Os parâmetros dos modelos cinéticos dos modelos Pseudo primeira ordem, Pseudo segunda ordem e Pseudo $n$ ordem foram calculados estão disponíveis na Tabela 2.

Tabela 2: Parâmetros calculados dos modelos cinéticos avaliados.

\begin{tabular}{|c|c|c|c|}
\hline MODELOS & PARÂMETROS & MODELOS & PARÂMETROS \\
\hline $\begin{array}{l}\text { Pseudo } \\
\text { primeira ordem }^{1}\end{array}$ & $\begin{array}{l}q_{\text {ecalc. }}\left(\mathrm{mg} \cdot \mathrm{g}^{-1}\right)=17,44 \pm 0,87 \\
k_{l}\left(\mathrm{~min}^{-1}\right)=0,22 \pm 0,09 \\
S_{R}^{2}\left(\mathrm{mg}^{2} \cdot \mathrm{g}^{-2}\right)=152,50 \\
R^{2}=0,647\end{array}$ & $\begin{array}{l}\text { Pseudo } \\
n \text { ordem }^{3}\end{array}$ & $\begin{array}{l}q_{\text {ecalc }}\left(\mathrm{mg} \cdot \mathrm{g}^{-1}\right)=20,83 \pm 1,26 \\
K_{n}\left(\mathrm{~g}^{\mathrm{n}-1} \cdot \mathrm{mg}^{1-\mathrm{n}} \cdot \mathrm{min}^{-1}\right)=0,00022 \pm 0,00020 \\
N=2,45 \pm 0,14 \\
S_{R}{ }^{2}\left(\mathrm{mg}^{2} \cdot \mathrm{g}^{-2}\right)=72,63 \\
R^{2}=0,820\end{array}$ \\
\hline $\begin{array}{l}\text { Pseudo } \\
\text { segunda ordem }\end{array}$ & $\begin{array}{l}q_{\text {ecalc. }}\left(\mathrm{mg} \cdot \mathrm{g}^{-1}\right)=18,17 \pm 0,81 \\
k_{2}\left(\mathrm{~g} \cdot \mathrm{mg}^{-1} \cdot \mathrm{min}^{-1}\right)=0,017 \pm 0,008 \\
S_{R}^{2}\left(\mathrm{mg}^{2} \cdot \mathrm{g}^{-2}\right)=102,41 \\
R^{2}=0,763\end{array}$ & Teste F & $\begin{array}{l}F_{c a l(1 / 2)}=1,49 \\
F_{c a l(1 / 3)}=2,10 \\
F_{c a l(2 / 3)}=1,41 \\
F_{t a b}=2,35\end{array}$ \\
\hline
\end{tabular}

A partir dos resultados do teste $F\left(F_{c a l}<F_{t a b}\right)$, considerando-se um nível de confiança de 95\% (Tabela 2), não existe diferença significativa entre os três modelos avaliados. No entanto, o modelo de pseudo $n$ ordem foi o que melhor se ajustou deixando menor resíduo (Tabela 2), sendo o valor de $n$ superior a 2, indicando que a maior taxa de adsorção ocorreu no inicio do processo, conforme [24].

Mesmo sem existir uma diferença significativa, nível de confiança de 95\%, entre os três modelos, o pseudo $n$ ordem representa um melhor ajuste aos dados experimentais, quando se comparado aos outros dois, indicando comportamento intermediário do processo de adsorção entre de pseudo primeira e pseudo segunda ordem, definindo, portanto, que mais de uma etapa é determinante para a velocidade de adsorção.

O modelo de difusão intrapartícula de Weber-Morris $\left(q_{t} v s . t^{1 / 2}\right)$ está apresentado na Figura 11. 


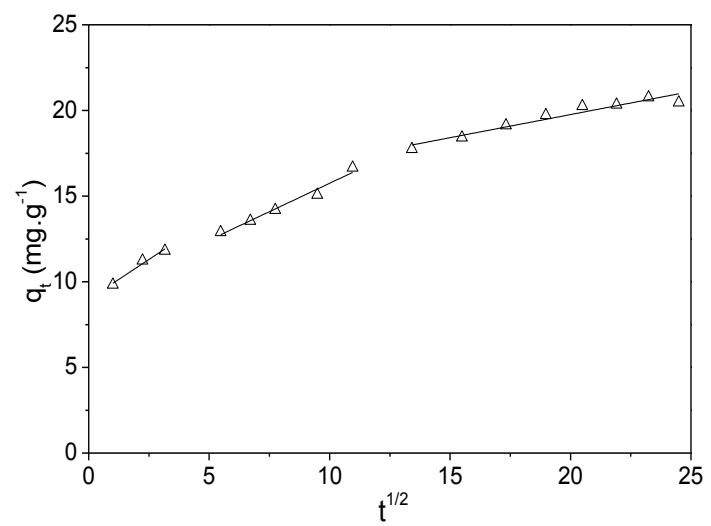

Figura 11: Modelo de difusão intrapartícula de Weber-Morris $\left(\mathrm{C}_{\mathrm{A}}\right)$.

Devido à multilinearidade dos dados e os gráficos não passarem pela origem, Figura 11, duas ou mais etapas controlam o processo, devendo-se avaliar cada região linear separadamente. A primeira faixa linear do gráfico sugere que a resistência à transferência de massa externa é significativa apenas no inicio do processo. Já a segunda faixa linear mostra um processo de adsorçãoé gradual, controlada pela difusão intrapartícula. A terceira faixa representa o estágio de equilíbrio conforme também encontrado por [4]. Os parâmetros do modelo de difusão intrapartícula de Weber Morris estão apresentados na Tabela 3.

Tabela 3: Parâmetros do modelo de difusão intrapartícula de Weber Morris.

\begin{tabular}{l|l|l|l}
\hline PARÂMETROS & $\begin{array}{l}\boldsymbol{k}_{\text {dif }} \\
\mathbf{m g} \cdot\left(\mathbf{g} \cdot \mathbf{m i n}^{\mathbf{0 . 5}} \mathbf{~}^{-1}\right.\end{array}$ & $\begin{array}{l}\boldsymbol{C} \\
\mathbf{( m g}^{-\mathbf{- 1}} \mathbf{)}\end{array}$ & $\boldsymbol{R}^{\mathbf{2}}$ \\
\hline Região 1 & $0,929 \pm 0,145$ & $8,979 \pm 0,336$ & 0,952 \\
Região 2 & $0,663 \pm 0,062$ & $9,115 \pm 0,514$ & 0,966 \\
Região 3 & $0,270 \pm 0,029$ & $14,361 \pm 0,583$ & 0,923 \\
\hline
\end{tabular}

Pode-se observar na Tabela 3, que a constante de difusão intrapartícula vai diminuindo com o decorrer do tempo, isto indica uma diminuição da difusão intrapartícula à medida que o fenol é adsorvido pelo carvão, a medida que há a diminuição da concentração de fenol na solução, conforme observado por [48].

$\mathrm{O}$ aumento nos valores da constante $\mathrm{C}$, Tabela 3, ocorre provavelmente devido ao aumento da camada limite na região, ocorrendo maior resistência à difusão.

As isotermas de adsorção e os ajustes não lineares dos modelos de Langmuir, Freundlich, FritzSchlunder e Redlich-Peterson estão apresentadas na Figura 12.

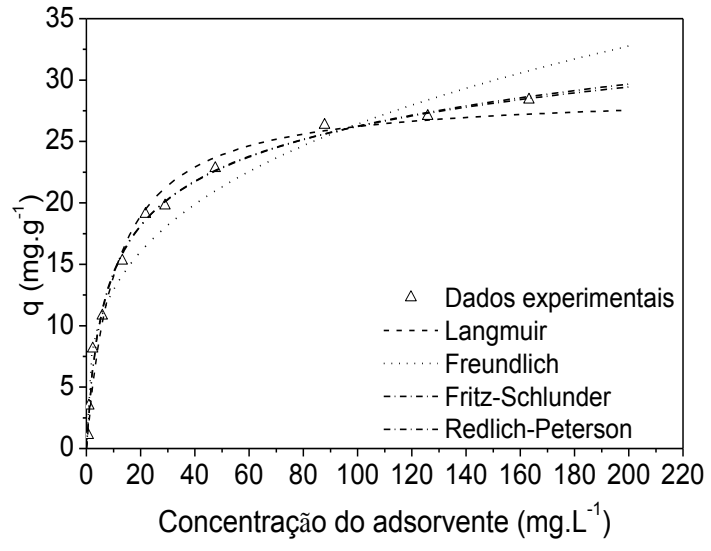

Figura 12: Isoterma de adsorção em sistema de banho finito, com os ajustes não lineares dos modelos $\mathrm{C}_{\mathrm{A}}$. $\mathrm{pH}=6,0 ; \mathrm{G}$. < 0,090 mm; V.A. $300 \mathrm{rpm} \mathrm{e} \mathrm{C}_{\mathrm{ads}}=4 \mathrm{~g} \cdot \mathrm{L}^{-1}$.

Constatou-se pela Figura 12, que os modelos de Fritz-Schlunder e Redlich-Peterson se sobrepõem aos 
dados experimentais. Os parâmetros dos modelos avaliados estão apresentados na Tabela 4.

Tabela 4: Parâmetros dos modelos de equilíbrio para adsorção de fenol a $25^{\circ} \mathrm{C}$.

\begin{tabular}{|c|c|c|c|c|}
\hline MODELOS & PARÂMETROS & MODELOS & PARÂMETROS & Teste F \\
\hline Langmuir $^{1}$ & $\begin{array}{l}q_{\max }=29,01 \pm 0,96 \\
B=0,094 \pm 0,013 \\
S_{R}^{2}\left(\mathrm{mg}^{2} \cdot \mathrm{g}^{-2}\right)=15,46 \\
R^{2}=0,98\end{array}$ & Freundlich $^{2}$ & $\begin{array}{l}K=6,33 \pm 0,90 \\
B=3,22 \pm 0,35 \\
S_{R}^{2}\left(\mathrm{mg}^{2} \cdot \mathrm{g}^{-2}\right)=50,88 \\
R^{2}=0,94\end{array}$ & $\begin{array}{l}F_{12}=3,29 \\
F_{13}=2,67 \\
F_{14}=2,64 \\
F_{23}=8,78 \\
F_{24}=9,11\end{array}$ \\
\hline $\begin{array}{l}\text { Fritz- } \\
\text { Schlunder }\end{array}$ & $\begin{array}{l}K_{F S}\left(\left(m g \cdot g^{-1}\right)\left(m g \cdot d m^{-3}\right)^{-b 1}\right)=4,63 \pm 0,99 \\
a\left(\left(m g \cdot d m^{-3}\right)^{-b 2}\right)=0,34 \pm 0,15 \\
b_{1}=1,09 \pm 0,30 \\
b_{2}=0,94 \pm 0,26 \\
S_{R}^{2}\left(\mathrm{mg}^{2} \cdot \mathrm{g}^{-2}\right)=5,79 \\
R^{2}=0,99\end{array}$ & $\begin{array}{l}\text { Redlich- } \\
\text { Peterson }^{4}\end{array}$ & $\begin{array}{l}A\left(\mathrm{~L} \cdot \mathrm{g}^{-1}\right)=4,59 \pm 0,86 \\
B\left(L \cdot m g^{-1}\right)^{g}=0,30 \pm 0,10 \\
G=0,87 \pm 0,03 \\
S_{R}^{2}\left(\mathrm{mg}^{2} \cdot \mathrm{g}^{-2}\right)=5,85 \\
R^{2}=0,99\end{array}$ & $\begin{array}{l}F_{34}=1,01 \\
F_{t a b}=3,18\end{array}$ \\
\hline
\end{tabular}

A partir dos resultados encontrados por meio do teste $F$, $\left(\mathrm{F}_{c a l}\right.$ (Tabela 4) $<\mathrm{F}_{t a b}(3,18)$ ), para um nível de 95\% de confiança não existe diferença significativa entre os modelos de Langmuir, Fritz-Schlunder e RedlichPeterson. Porém o modelo de Langmuir apresentou uma variância $\left(S_{R}{ }^{2}\right) 3$ vezes maior que os modelos FritzSchlunder e Redlich-Peterson. Entretanto, ocorreu uma diferença significativa quando os modelos foram comparados ao de Freundlich. Este modelo também foi o que apresentou maior variância $\left(S_{R}{ }^{2}\right)$, não sendo assim, o mais adequado para representar os dados experimentais.

Dentre os quatro modelos avaliados, os que melhor se ajustaram aos dados experimentais, foram os de Fritz-Schlunder e Redlich-Peterson, de três e quatro parâmetros, respectivamente. Apresentaram o $\mathrm{R}^{2}>0,99$ e menor valor de variância $\left(\mathrm{S}_{\mathrm{R}}{ }^{2}\right)$ com relação aos de Lagmuir e ao de Freundlich. na Tabela 5 .

Uma comparação dos resultados obtidos neste trabalho e aos encontrados na literatura estão apresenta

Tabela 5: Comparação dos resultados deste trabalho aos encontrados na literatura para adsorção de fenol.

\begin{tabular}{|c|c|c|c|c|c|c|}
\hline PRECURSOR & ATIVAÇÃO & $\begin{array}{l}\text { D.A } \\
\left(\mathrm{g} \cdot \mathrm{L}^{-1}\right)\end{array}$ & $\begin{array}{l}\mathrm{C}_{0} \\
\left(\mathrm{mg} \cdot \mathrm{L}^{-1}\right)\end{array}$ & $\begin{array}{l}\text { EQUILÍBRIO } \\
(\mathrm{min})\end{array}$ & $\begin{array}{l}\boldsymbol{q}_{\max } \\
\left(\mathbf{m g} \cdot \mathbf{g}^{-1}\right)\end{array}$ & REFERÊNCIA \\
\hline $\begin{array}{l}\text { Resíduos } \\
\text { agrícolas }\end{array}$ & $\begin{array}{l}\text { Calc. } 900{ }^{\circ} \mathrm{C}(2 \mathrm{~h}) \\
\mathrm{CO}_{2} 900^{\circ} \mathrm{C}(2 \mathrm{~h})\end{array}$ & 8 & 6800 & 4320 & 23,8 & {$[14]$} \\
\hline Casca de coco & Calc. $600^{\circ} \mathrm{C}(1 \mathrm{~h})$ & 2 & 74 & 1800 & 34 & {$[16]$} \\
\hline $\begin{array}{l}\text { Caroço de } \\
\text { abacate }\end{array}$ & $\begin{array}{l}\mathrm{N}_{2}\left(800^{\circ} \mathrm{C} ; 1 \mathrm{~h}\right) \\
\mathrm{CO}_{2}\left(900^{\circ} \mathrm{C} ; 3 \mathrm{~h}\right)\end{array}$ & 2 & 600 & 60 & 90 & {$[15]$} \\
\hline $\begin{array}{l}\text { Resíduo de } \\
\text { Eucalyptus }\end{array}$ & $\begin{array}{l}\mathrm{N}_{2}\left(500^{\circ} \mathrm{C} ; 2 \mathrm{~h}\right) \\
\operatorname{Ar}\left(400^{\circ} \mathrm{C} ; 2 \mathrm{~h}\right)\end{array}$ & 1 & 500 & 600 & 64 & {$[17]$} \\
\hline $\begin{array}{l}\text { Casca de } \\
\text { amendoim }\end{array}$ & $\begin{array}{l}\mathrm{N}_{2}\left(600^{\circ} \mathrm{C} ; 1 \mathrm{~h}\right) \\
\mathrm{CO}_{2}\left(600^{\circ} \mathrm{C} ; 1 \mathrm{~h}\right)\end{array}$ & 4 & 300 & 120 & 29,01 & \begin{tabular}{|l} 
Esse \\
Trabalho
\end{tabular} \\
\hline
\end{tabular}

De acordo com os resultados apresentados na Tabela 5, pode-se verificar que o carvão da casca de amendoim ativado fisicamente com $\mathrm{CO}_{2}$ possui boa eficiência quando comparado aos resultados de outros carvões encontrados na literatura. 
O processo de ativação demonstrou ser uma alternativa atraente, de modo geral, devido a menor demanda de energia por apresentar temperaturas e tempo de ativação mais amenos. Além disso, a concentração inicial e tempo de equilíbrio são relativamente baixos, quando comparados a maioria dos trabalhos.

É importante destacar a grande quantidade de casca de amendoim residual produzida no Brasil. Tal resíduo pode ser utilizado na preparação de adsorvente com aplicação industrial. Compatibilizando as questões técnicas e ambientais, pois a partir de um resíduo agroindustrial, pode ser preparado um adsorvente eficiente no processo de remoção de contaminante de efluentes industriais.

\section{CONCLUSÕES}

A partir dos resultados apresentados nesse trabalho, constatou-se que a casca de amendoim é um resíduo viável para a produção de adsorvente.

Após o processo de carbonização e ativação da casca de amendoim, o $\mathrm{C}_{\mathrm{A}}$ apresentou características como o aumento da área superficial e do volume de poros, a presença de uma maior quantidade de grupos funcionais básicos, maior estabilidade térmica, maior quantidade de carga superficial positiva e reduzida cristalinidade, que potencializam a capacidade de adsorção do material.

No estudo cinético, o modelo de pseudo $n$ ordem foi o que melhor se ajustou aos dados experimentais indicando uma elevada taxa de adsorção inicial. O modelo de Weber-Morris indicou que o processo é controlado por mais de uma etapa, incluindo difusão intrapartícula.

A capacidade adsortiva máxima obtida no estudo de equilíbrio foi de $29,01 \pm 0.96 \mathrm{mg} \cdot \mathrm{g}^{-1}$, tendo os modelos de Redlich-Peterson e Fritz-Schlunder um melhor ajuste aos dados experimentais.

Os resultados demonstraram a capacidade técnica do adsorvente para a remoção de fenol em solução aquosa. É importante ressaltar que o carvão foi preparado a partir de resíduo agroindustrial, que possui alta disponibilidade e é oriundo de recursos renováveis.

\section{AGRADECIMENTOS}

Ao Centro de Tecnologias Estratégicas do Nordeste (CETENE), ao Laboratório de Tecnologias Limpas (LATECLIM)/DEQ/UFPE, ao NUQAAPE/FACEPE, à Agência Nacional do Petróleo, Gás Natural e Biocombustíveis - ANP e à Financiadora de Estudos e Projetos - FINEP - por meio do Programa de Recursos Humanos da ANP para o Setor de Petróleo e Gás - PRH-ANP/MCT, em particular ao PRH 28, do Departamento de Engenharia Química, Centro de Tecnologia e Geociências da UFPE.

\section{BIBLIOGRAFIA}

[1] MARIANO, J. B., Impactos ambientais do refino de petróleo, 1 ed., Rio de Janeiro, Interciência, 2005.

[2] KULKARNI, S. J., TAPRE, R. W., PATIL, S. V., et al., "Adsorption of Phenol from Wastewater in Fluidized Bed Using Coconut Shell Activated Carbon”, Procedia Engineering, v. 51, pp. 300-307, 2013.

[3] WU, Z., ZHOU, M. "Partial degradation of phenol by advanced electrochemical oxidation process", Scence and Technoogyl, v. 35, n. 13, pp. 2698-2703, Mai, 2001.

[4] FIERRO, V., TORNE'-FERNÁNDEZ, V., MONTANÉ, D., et al., “Adsorption of phenol onto activated carbons having different textural and surface properties", Microporous and Mesoporous Materials, v. 111, n. 3, pp. 276-284, Abr, 2008.

[5] ESTRADA-ARRIAGA, E. D., ZEPEDA-AVILES, J. A., GARCÍA-SÁNCHEZ, L. "Post-treatment of real oil refinery effluent with high concentrations of phenols using photo-ferrioxalate and Fenton's reactions with membrane process step", Chemical Engineering Journal, v. 285, pp. 508-516, Fev, 2016.

[6] ACIKGOZ, E., OZCAN, B., "Phenol biodegradation by halophilic archaea", International Biodeterioration and Biodegradation, v. 107, pp. 140-146, Fev, 2016.

[7] ZHANG, D., HUO, P., LIU, W., "Behavior of phenol adsorption on thermal modified activated carbon", Chinese Journal of Chemical Engineering, v. 24, n. 4, pp. 446-452, Abr, 2016.

[8] ANGIN, D. "Utilization of activated carbon produced from fruit juice industry solid waste for the adsorption of Yellow 18 from aqueous solutions”, Bioresource Technology, v.168, pp. 259-266, Set, 2014.

[9] DALlAGO, R. M., SMANIOTTO A., "Resíduos sólidos de curtumes como adsorventes para a remoção de corantes em meio aquoso", Química Nova, v. 28, pp. 433-437, Mai, 2005. 
[10] AVELAR, F. F., BIANCHI, M. L., GONÇALVES, M., et al., "The use of piassava fibers (Attaleafunifera) in the preparation of activated carbon", Bioresource Technology, v. 101, n. 12, pp. 4639-4645, Jun, 2010.

[11] VIEIRA, A. P., SANTANA, S. A. A., BEZERRA, C. W. B., et al., "Epicarp and Mesocarp of Babassu (Orbignyaspeciosa): Characterization and Application in Copper Phtalocyanine Dye Removal", Journal of the Brazilian Chemical Society, v. 22, n. 1, pp. 21-20, Jan, 2011.

[12] MARÍA, S. C., CARLOS. A. P., LEIRE. Z., et al., "A comparison of physical activation of carbon xerogels with carbon dioxide with chemical activation using hydroxides", Carbon, v. 48, pp. 3157-3168, Mai, 2010.

[13] MACiÁ-AGUlló, J. A., MOORE. B. C., CAZORLA-AMORÓS. D., et al., "Activation of coal tar pitch carbon fibres: Physical activation vs. chemical activation”, Carbon, v. 42, pp. 1361-1364, Dez, 2004.

[14] SEKIRIFA M. L., HADJ-MAHAMMED M., PALLIER S., et al., "Preparation and characterization of an activated carbon from a date stones variety by physical activation with carbon dioxide", Journal of Analytical and Applied Pyrolysis, v.99, pp.155-160, Jan, 2013.

[15] RODRIGUES, L. A., SILVA, M. L. C. P., ALVARES-MENDES, M. O., et al., "Phenol removal from aqueous solution by activated carbon produced from avocado kernel seeds", Chemical Engineering Journal, v. 174, n. 1, pp.49-57, Out, 2011.

[16] SINGH K.P., MALIK A., SINHA S., et al., "Liquid-phase adsorption of phenols using activated carbons derived from agricultural waste material”, Journal of Hazardous Materials, v. 150, pp. 626-641, Fev, 2008.

[17] TANCREDI, N., MEDERO, N., MÖLLER, F., et al., "Phenol adsorption onto powdered and granular activated carbon, prepared from Eucalyptus wood", Journal of Colloid and Interface Science, v. 279, pp.357363, Nov, 2004.

[18] COUTO, G. M., DESSIMONI, A, L. A., BIANCHI, M. L., et al., "Use of sawdust Eucalyptus sp. in the preparation of activated carbons”, Ciência e Agrotecnologia, v. 36, pp. 69-77, Fev, 2012.

[19] CONAB - Campanhia Nacional de Abastecimento. Acompanhamento da Safra Brasileira. Grãos. v.2 Safra 2014/15, n.5 - Quinto Levantamento, 2015.

[20] GATANI, M. P., ARGUELlO, R., MEDINA, J. C., et al., "Viabilidade técnica de produção e propriedades de painéis de partículas de casca de amendoim”, Revista Materia, v.18, pp. 1286-1293, Jun, 2013.

[21] REGALBUTO, J., Catalyst preparation: Science and engineering, 1 ed. New York, CRC Press, 2016.

[22] BARROS NETO, B., SCARMINIO, I. S., BRUNS, R. E., Como Fazer Experimentos: pesquisa e desenvolvimento na ciência e na indústria, 2 ed., Campinas, Unicamp, 2007.

[23] MIMURA, A. M. S., VIEIRA, T. V. A., MARTELLI, P.B, et al., "Aplicação da casca de arroz na adsorção dos íons $\mathrm{Cu}^{+2}, \mathrm{Al}^{+3}, \mathrm{Ni}^{+2}$ e Zn ${ }^{+2}$, Química Nova, v. 33, n. 6, pp.1279-1284, Jun, 2010.

[24] TSENG, R-L, WU, P-H, WUC, F-C, et al., "A convenient method to determine kinetic parameters of adsorption processes by nonlinear regression of pseudo-nth-order equation", Chemical Engineering Journal, v. 237, pp. 153-161, Fev, 2014.

[25] WEBER, W. J., MORRIS, J. C., "Kinetics of adsorption on carbon from solution", Journal of Sanitary Engineering Division ASCE, v. 89, n. 2, pp. 31-60, 1963.

[26] GIRALDO, L., MORENO-PIRAJÁN, J. C., "Study of adsorption of phenol on activated carbons obtained from egg shells", Journal of Analytical and Applied Pyrolysis, v. 106, pp. 41-47, Mar, 2014.

[27] WANYONYI. W. C., ONYARI, J. M., SHIUNDU, P. M., "Adsorption of congo red dye from aqueous solutions using roots of Eichhornia crassipes: kinetic and equilibrium studies", Energy Procedia, v. 50, pp. 862-869, 2014

[28] NASCIMENTO, R. F., LIMA, A. C. A., VIDAL, C. B., et al., Adsorção: aspectos teóricos e aplicações ambientais, 1 ed., Fortaleza, UFC, 2014.

[29] FRITZ, W., SCHLUNDER, E. U., "Simultaneous adsorption equilibria of organic solutes in dilute aqueous solution on activated carbon", Chemical Engineering Science, v. 29, n. 5, pp. 1279-1282, Mai, 1974.

[30] MONTGOMERY, D. C., Introdução ao controle estatístico da qualidade, 4 ed., Tempe, LTC, 2012.

[31] THOMMES, M., KANEKO, K., NEIMARK, A. V., et al., "Physisorption of gases, with special reference to the evaluation of surface area and pore size distribution (IUPAC Technical Report)", Pure and Applied Chemistry, v. 87, n. 10, pp. 1051-1069, Out, 2015. 
[32] REINOSO, F., LAHAYE, J., EHRBURGER, P., Fundamental issues in control of carbon gasification reactivity, 1 ed., Netherlands, Springer, 1991.

[33] HAN, Y., BOATENG, A. A., QI, P. X., et al., "Heavy metal and phenol adsorptive properties of biochars from pyrolyzed switchgrass and woody biomass in correlation with surface properties", Journal of Environmental Management, v.118, pp.196-204, Fev, 2013.

[34] BORGES, W. M. S., ARANTES, A. C. C., CASTRO, G. M. M., et al., "Produção, caracterização e avaliação da capacidade adsortiva de carvões ativado em forma de briquete", Matéria, v. 21, pp. 930-942, Dez, 2016.

[35] LOWELL, S., SHIELDS, J. E., THOMAS, M.A., et al., Characterization of Porous Solids and Powders: Surface Area, Pore Size and Density, 1 ed., Dordrecht, Springer, 2004.

[36] ZHONG, Z-Y., YANGA, Q., LI, X-M., et al., "Preparation of peanut hull-based activated carbon by microwave-induced phosphoric acid activation and its application in Remazol Brilliant Blue R adsorption", Industrial Crops and Products, v. 37, n. 1, p.178- 185, Mai, 2012.

[37] SCHETINO JÚNIOR, M. A., FREITASK, J. C. C., CUNHA, A. G., et al., "Preparação e caracterização de carvão ativado quimicamente a partir da casca de arroz", Química nova, v. 30, n. 7, pp. 1663-1668, Ago, 2007.

[38] GUILARDUCI, V. V. S., MESQUITA, J. P., MARTELLI, P. B., et al., "Adsorção de fenol sobre carvão ativado em meio alcalino", Química Nova, v. 29, n. 6, pp. 1226-1232, Jun. 2006.

[39] MARTINS, A. F., CARDOSO, A. L., STAHL, J. A., et al., "Low temperature conversion of rice husks, eucalyptus sawdust and peach stones for the production of carbon-like adsorbent", Bioresource technology, v. 98, n. 5, pp. 1095-1100, Mar, 2007.

[40] TONGPOOTHORN, W., SRIUTTHA, M., HOMCHAN, P., et al., "Preparation of activated carbon derived from Jatrophacurcasfruitshell by simple thermo-chemical activation andcharacterization of their physico-chemical properties", Chemical engineering research and design, v. 89, n.X, pp. 335-340, Mar, 2011.

[41] SOLOMONS, T. W. G., FRYHLE, C. B., Química Orgânica, 9 ed. Rio de Janeiro, LTC, 2009.

[42] YAGMUR, E., OZMAK, M., AKTAS, Z. "A novel method for production of activated carbon from waste tea by chemical activation with microwave energy", Fuel, v. 87, n. 15, pp. 3278-3285, Nov, 2008.

[43] DABROWSKI, A., PODKOSCIELNY. P., HUBICKI. Z., et al., "Adsorption of phenolic compounds by activated carbon - A critical review", Chemosphere, v. 58, n. 8, pp. 1049-1070, Sep. 2005.

[44] MATTSON, J. S.; MARK, H. B., Activated Carbon, X ed.,New York, Marcel Dekker, 1971.

[45] SCHWANTES, D., GONÇALVES JR, A. C., CASARIN, J., et al., "Removal of Cr (III) from contaminated water using industrial waste of the cassava as natural adsorbents", African Journal of Agricultural Research, v. 10, pp. 4241-4251, Nov, 2015.

[46] SAHA, P., CHOWDHURY, S., GUPTA, S., et al., "Insight into adsorption equilibrium, kinetics and thermodynamics of Malachite Green onto clayey soil of Indian origin", Journal Chemical Engineering, v. 165 , n. 3, pp. 874-882, Dez, 2010.

[47] SCHIMPF, S., LOUIS, C., CLAUS, P., "Ni/ $\mathrm{SiO}_{2}$ catalysts prepared with ethylenediamine nickel precursors: Influence of the pretreatment on the catalytic properties in glucose hydrogenation", Applied Catalysis A: General, v. 318, pp. 45-53, Fev, 2007.

[48] ARTHY M., SARAVANAKUMAR M. P., "Isotherm modeling, kinetic study and optimization of batch parameters for effective removal of Acid Blue 45 using tannery waste", Journal of Molecular Liquids, v. 187, pp. 189-200, Nov, 2013. 